\title{
BMJ Open Quality Improving the quality of insulin prescribing for people with diabetes being discharged from hospital
}

\author{
Amie Bain, ${ }^{\oplus 1,2}$ Jon Silcock, ${ }^{3}$ Sallianne Kavanagh, ${ }^{2}$ Gemma Quinn, ${ }^{3}$ Ines Fonseca ${ }^{2}$
}

To cite: Bain A, Silcock J, Kavanagh S, et al. Improving the quality of insulin prescribing for people with diabetes being discharged from hospital. BMJ Open Quality 2019;8:e000655. doi:10.1136/ bmjoq-2019-000655

Received 8 February 2019 Revised 6 June 2019 Accepted 6 August 2019

\section{Check for updates}

(c) Author(s) (or their employer(s)) 2019. Re-use permitted under CC BY-NC. No commercial re-use. See rights and permissions. Published by BMJ.

${ }^{1}$ School of Applied Sciences, University of Huddersfield, Huddersfield, UK

${ }^{2}$ Department of Pharmacy, Sheffield Teaching Hospitals NHS Foundation Trust, Sheffield, UK

${ }^{3}$ School of Pharmacy and Medical Sciences, University of Bradford Faculty of Life Sciences, Bradford, UK

Correspondence to Amie Bain; a.bain@hud.ac.uk

\section{ABSTRACT}

Medication errors involving insulin in hospital are common, and may be particularly problematic at the point of transfer of care. Our aim was to improve the safety of insulin prescribing on discharge from hospital using a continuous improvement methodology involving cycles of iterative change. A multidisciplinary project team formulated locally tailored insulin discharge prescribing guidance. After baseline data collection, three 'plan-do-study-act' cycles were undertaken over a 3-week period (September/ October 2018) to introduce the guidelines and improve the quality of discharge prescriptions from one diabetes ward at the hospital. Discharge prescriptions involving insulin from the ward during Monday to Friday of each week were examined, and their adherence to the guidance measured. After the introduction of the guidelines in the form of a poster, and later a checklist, the adherence to guidelines rose from an average of $50 \%$ to $99 \%$. Qualitative data suggested that although it took pharmacists slightly longer to clinically verify discharge prescriptions, the interventions resulted in a clear and helpful reminder to help improve discharge quality for the benefit of patient safety. This project highlights that small iterative changes made by a multidisciplinary project team can result in improvement of insulin discharge prescription quality. The sustainability and scale of the intervention may be improved by its integration into the electronic prescribing system so that all users may access and refer to the guidance when prescribing insulin for patients at the point of discharge.

\section{PROBLEM}

Insulin is a high-risk, critical medicine widely used in the treatment of diabetes mellitus, where inappropriate use can lead to poor glycaemic control, patient harm or even death. ${ }^{2}$ Errors involving insulin remain common, despite various local and national initiatives to decrease their number, and are particularly prevalent in hospital at the pointof-care transfer. $^{3-7}$

Sheffield Teaching Hospitals NHS Foundation Trust (STHFT) is one of the largest and busiest National Health Service (NHS) hospital trusts in England, operating across five hospitals and 40 community sites. Over 2.3 million patients a year are seen at STHFT, $15 \%$ of whom have a diagnosis of diabetes. Like many other large teaching hospitals, staffing pressures and busy working environments may jeopardise safe and accurate insulin prescribing, particularly due to the complex nature of the medication and prescribing processes. The prescribing of insulin at the trust has been subject to the phased introduction of inpatient electronic prescribing and medicines administration (EPMA) over the past year, which has further complicated the prescribing process locally.

Although a variety of insulin safety measures have been introduced at STHFT, such as e-learning modules, specialist diabetes link nurses, dedicated insulin charts, specific foundation doctor training and EPMA, there have been consistently high levels of insulin errors being reported locally, and insulin is still one of the top medications implicated in medication errors at the hospital.

Clinical audits and previous research into insulin errors at the hospital showed that between $46 \%$ and $86 \%$ of patients prescribed insulin experience at least one insulin error during their stay; and despite the use of electronic discharge summaries for a number of years, insulin errors persist and are considered preventable by staff. ${ }^{6-8}$

In order to further explore insulin errors during EPMA implementation at STHFT, the diabetes pharmacy team conducted a 6-month, retrospective thematic analysis of local medication safety incident reports involving insulin errors (online supplementary figure S1, table S1). In light of the recently reported errors, it was felt that systems and processes could be further optimised for the benefit of patient safety, particularly in the period of initial implementation and development of EPMA. The results were discussed with the diabetes inpatient service improvement team at the hospital, and due to the prevalence and nature of reported errors at discharge, it was agreed that the quality of insulin discharge prescriptions should be the focus of quality improvement (QI) efforts. 
A multidisciplinary project team, led by a pharmacist academic practitioner, was established from members of the diabetes inpatient service improvement team and included consultant diabetologists, diabetes inpatient specialist nurses, and diabetes pharmacists and pharmacy technicians. After examination of both previous and existing systems and measures to promote the safe prescribing of insulin at the point of discharge (both at STHFT and nationally), the project team decided to develop a set of consensus-derived discharge prescribing guidelines concerning insulin (online supplementary table S2). These were created in line with national guidance such as the Joint British Diabetes Societies' Guidelines on discharge planning ${ }^{9}$ but tailored to the local context.

\section{BACKGROUND}

Medication errors have previously been described as 'common' at the point of discharge,${ }^{510}$ and can include the unintended omission of medication, continuation/ re-prescribing of an intentionally stopped medication, or an error in medication dose, frequency or formulation. ${ }^{11}$ If discharge prescription errors are not rectified, they may persist in the community, thus posing an even greater threat to patient safety. ${ }^{12}$

The implementation of electronic communication systems and medicines reconciliation processes have been recommended to reduce medication errors at the point of discharge. ${ }^{13-15}$ The impact of medicines reconciliation on insulin prescribing quality was previously studied at the trust with positive results, ${ }^{7}$ but the impact of this process at the point of discharge has not yet been examined locally. Although electronic discharge summaries have been in use for a number of years at STHFT, insulin discharge prescriptions were often found to contain insufficient information for general practitioners and community care providers. ${ }^{6}$

The impact of insulin prescribing errors at the point of discharge for people using insulin may be particularly severe due to the increased risk associated with its use. Previously, high volumes of insulin-related patient safety incident reports prompted the National Patient Safety Agency (now part of NHS Improvement) to issue a rapid response alert to NHS providers in 2010, instructing them to make changes to the way insulin was prescribed. ${ }^{16}$ These included writing 'units' instead of 'u' or 'iu', the provision of training for all healthcare staff involved with insulin and the introduction of an 'Insulin Passport', where contemporaneous insulin details can be kept with the patient throughout their care journey. ${ }^{17}$

Despite this, the National Diabetes Inpatient Audit has shown consistently high levels of insulin errors since 2011; in 2017, 4 out of 10 inpatients with insulin-treated diabetes experienced an insulin error in England and Wales. ${ }^{4}$ Despite the promotion of EPMA as a strategy to reduce insulin errors, insulin prescribing errors are only slightly less likely to occur at trusts using EPMA (17.2\% cf $18.9 \%$ ), and often persist despite its introduction. ${ }^{4} 8$ The success of the Insulin Passport has also been limited, prompting the discontinuation of their routine use at STHFT as well as elsewhere in the UK. ${ }^{18}$

It is evident that erroneous insulin prescribing is still prevalent and problematic, particularly at the point of discharge. A recent systematic review on insulin prescribing interventions concluded that implementing strategies that are sensitive to local context and designed to increase adherence to insulin prescribing guidelines are associated with a reduction in prescribing errors. ${ }^{19}$

Approaches to reducing risk with insulin prescribing at discharge are variable and subject to differences in guidance interpretation and local influences. ${ }^{20}$ Previous QI interventions in the UK have involved root cause analysis, the introduction of a regional inpatient drug chart, insulin safety posters, prompts, educational tools and comprehensive discharge checklists. ${ }^{21-25}$ A recent failure modes, effects and criticality analysis identified a number of factors involved in the failures of the discharge process for patients using insulin, including variability in delivery of diabetes education and training, care coordination and medication prescribing patterns. ${ }^{26}$ The resulting recommendations to improve prescribing and reduce medication errors included implementing institution-specific, validated, consensus-derived discharge guidelines which could be embedded within an electronic health record decision support tool.

In light of the recent problems with insulin prescribing at discharge at STHFT, as well the wider issues of insulin prescribing safety both in the UK and globally, this project focused on improving the quality of electronic insulin prescribing at the point of discharge from hospital through the use of continuous improvement methodology.

\section{Patient and public involvement}

Although not directly involved in the design of the interventions included in this project, the STHFT diabetes patient public involvement group was consulted and gave their support to the idea and need for the insulin prescribing safety project, which was informed by their concerns around communication issues and not feeling in control of their insulin in hospital. The group expressed interest in disseminating findings and involvement in the ongoing wider research in this area at the Trust.

\section{MEASUREMENT}

In order to investigate the extent of the current problem, baseline data were retrospectively collected from the 10 most recent electronic patient discharge prescriptions involving insulin from one diabetes ward during 1 week (Monday-Friday) by a single pharmacist. The ward was chosen in light of its comparatively high volume of reported insulin errors and greater proportion of patients being prescribed insulin compared with other wards. A proforma was used to record completed discharge 
prescription details and the degree of adherence to insulin prescribing guidance (online supplementary table S3). Degree of adherence to prescription aspects of the guidance was scored for each area and the result was inputted into a run chart to detect any common cause variation.

Two of the 10 discharged patients examined at baseline were prescribed two insulin products on discharge, making the total number of insulin prescriptions measured at baseline equal to 12. None of the discharge prescriptions complied fully with the guidance measures. One patient's insulin (two products) was completely omitted from the discharge prescription (thereby achieving $0 \%$ compliance). There were four prescriptions for 'when required' correctional insulin, two of which were judged to be unnecessary at the point of discharge (ie, 'when required' insulin had not been prescribed on admission nor for at least 7 days preceding discharge) and the other two did not contain clear instructions for use. Only three discharge prescriptions mentioned arrangements for insulin administration at home. Seventy per cent of prescribed insulin contained sufficient information about the product, dose, frequency, device and time and $44 \%$ explicitly described the changes made to insulin since admission. Results from baseline data collection therefore confirmed that insulin prescribing quality at discharge required improvement.

Although the use of the overall scoring system used to measure improvement involved making quality judgements and did not focus on adherence to one particular measure of improvement, it did allow for a more general assessment of the quality of insulin prescriptions on discharge. As such, the team decided to use the same proforma to collect data chronologically throughout the project, which ran over the following 3 weeks.

Throughout the project, data were retrospectively collected using the hospital's EPMA system by the same individual at the end of the week to ensure reliable and complete collection that did not impede on workflow in real time. In order to increase rigour and limit bias, a second pharmacist independently reviewed the data collection tool and the quality judgements made using the scoring system. No identifiable patient details were recorded on the proforma. All data were anonymised and kept confidentially on a secure personal drive only available via a hospital-networked computer.

\section{DESIGN}

The baseline data relating to insulin discharge prescriptions were discussed within the multidisciplinary project team, and it was agreed that an intervention to improve the quality of discharges was needed. The team communicated frequently via email and members of the team met in person at various intervals (eg, some members met weekly, others monthly) to discuss the project progress. The members of the team were key stakeholders in the insulin safety and diabetes care agenda and were therefore ideally placed to help the sustainability of the project.

Insulin discharge guidelines were developed by project team consensus and posters were designed to display the guidance on the selected ward areas (figure 1). All members of the project team worked with staff on the ward to inform the design of interventions, as well as facilitatetheir implementation and development throughout the project.

The posters were developed through several iterations by the project team leader, informed by feedback from all members of the project team, over a 2-week period before being introduced on the ward. Posters were displayed, with consent from ward staff, around workstations where electronic prescribing occurred. In order to increase the impact of the poster on improving patient safety, it was felt that the poster should include guidance on all relevant and problematic areas of the discharge process. The poster would therefore be a more useful reference for staff undertaking patient discharges, despite the measurement of adherence to only specific elements of guidance.

Posters were designed to be engaging, readable, relevant and understandable, with clear navigation planes and drawing on the principals of the national 'Think Glucose' campaign. ${ }^{27}{ }^{28}$ Feedback on the posters and guidance was obtained via informal qualitative interviews and helped to inform future iterations of the project.

An integrated approach to introducing the guidelines was taken in order to help engage the intended audience and improve knowledge transfer as well as a change in attitudes and behaviour. ${ }^{29}$ This involved early reciprocal dialogue with healthcare professionals on the ward, as well as engagement of senior staff. The early involvement of the trust-wide diabetes team, as well as dedicated educational sessions, inclusion in the hospital's diabetes educational packages and integration into the EPMA system helped to enable scale and sustainability of the intervention.

\section{STRATEGY}

Our aim was to improve the quality of insulin discharge prescriptions on one diabetes ward so that all prescriptions complied with the new insulin prescribing guidance within 3 weeks. Our main outcome measure was the quality of insulin discharge prescriptions being issued from the diabetes ward, which was assessed by a combination of quality judgements and measures, resulting in an overall percentage score for each prescription. The data were measured continuously and remotely over a total period of 4 weeks, with all discharges involving insulin from the ward (Monday-Friday) being included. We undertook three 'plan-do-study-act' (PDSA) test cycles over the 3-week intervention period.

\section{PDSA cycle 1}

Our initial intervention was to introduce the posters on the ward areas and explain the project to the nursing, 

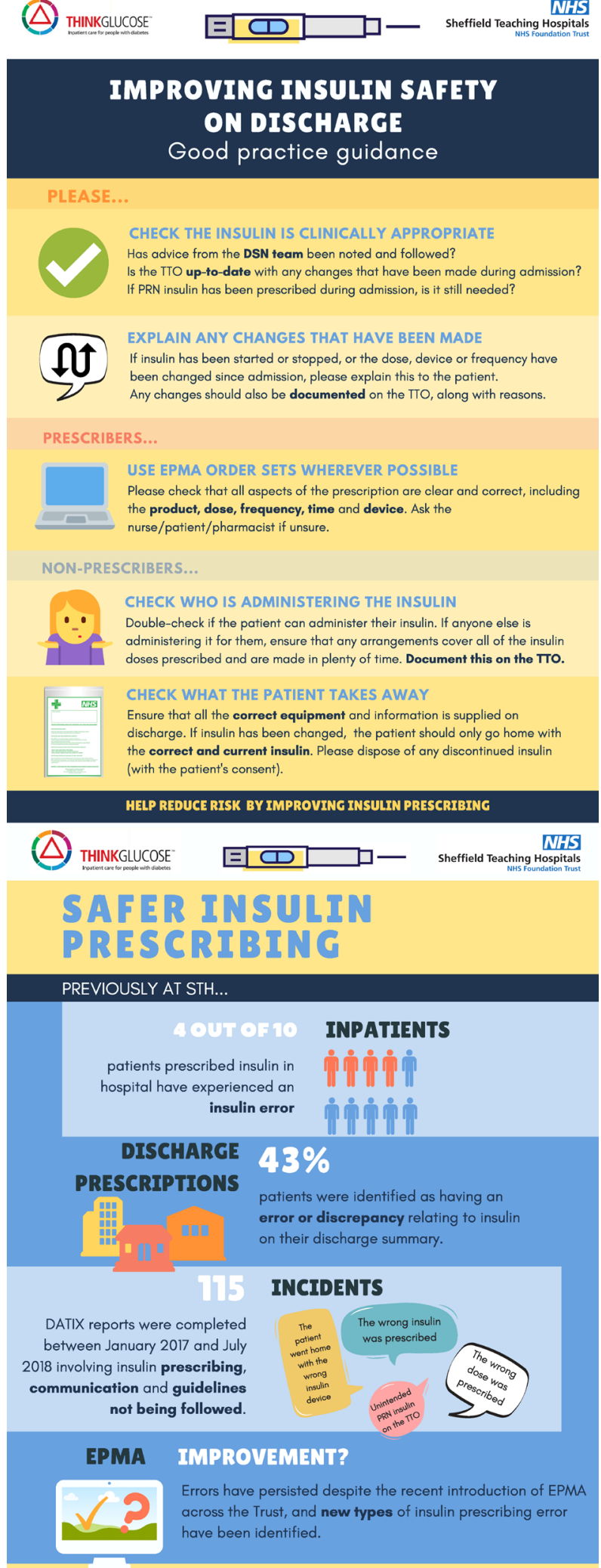

HELP REDUCE RISK BY IMPROVING INSULIN PRESCRIBING Please refer to the new good practice guidance regarding insulin prescribing at discharge

Figure 1 Insulin safety poster: 'improving insulin safety on discharge' (A) and project rationale poster, 'safer insulin prescribing' $(B)$ introduced to the ward in plan-do-study-act (PDSA) cycle 1. EPMA, electronic prescribing and medicines administration. medical and pharmacy staff on the ward over the course of one afternoon. Ward staff were positive about the need for the guidance and the look of the posters, and agreed where the posters should be displayed on ward areas so that they could be referred to when discharging patients. This resulted in an increase of adherence to guidance by an average of $22 \%$. Feedback received indicated that the posters were a helpful prompt for all ward staff involved in discharging patients using insulin.

\section{PDSA cycle 2}

The particular lack of documentation regarding insulin administration arrangements on discharge was evident during continuous data collection. On further investigation, it was found that, although the diabetes management and monitoring chart has a designated section on insulin self-administration status, this was very rarely completed. As a result, insulin administration information was not easily accessible on the EPMA system. The team decided if self-administration status was included as a 'free-text' prescription item as part of the medicines reconciliation process on admission, staff would be clearly informed from the point of admission. As others have shown, this should prompt consideration of self-management during admission and promoting insulin safety, ${ }^{30}$ and allow early referral to the diabetes specialist nurse if district nurse input was required for insulin administration at home. As a result of this small adjustment to the medicines reconciliation process on admission, compliance with the overall guidance increased by a further $20 \%$.

\section{PDSA cycle 3}

After discussion with the ward team, who felt clearer, tailored instruction was needed for particular professional groups (eg, nurses, pharmacists, doctors), a checklist was created for nurses to actively use when discharging patients with insulin (figure 2). Checklists have been shown to be useful for improving patient safety in various clinical settings by strengthening compliance with guidelines, improving human factors and reducing the incidence of adverse events. ${ }^{31}$ An iterative process of checklist design was employed with consultation with the team, and interestingly, highlighted slight variations in practice that could be addressed to provide a more consistent approach to patient safety. Checklists were designed to be clear, simple and further prompt a systematic process to ensuring good practice when discharging patients with insulin. Although 'checklist compliance' was not measured due to its poor reflection of task execution, ${ }^{32}$ after their introduction, there was an additional $7 \%$ improvement in overall guideline compliance.

\section{RESULTS}

One-week baseline measurement of 10 patients (12 insulin prescriptions) showed normal variation, and after each intervention, a general upward trend was observed. After three consecutive PDSA cycles involving a further 16 patients (20 insulin prescriptions) over the following 
THINKGLUCOSE" $\quad$ E

- PILOT -

\section{INSULIN DISCHARGE CHECKLIST}

For nurses

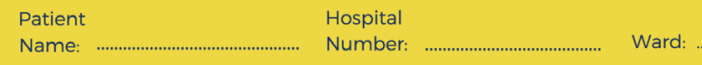

The patient is safe for discharge and DSN advice has been followed (where given)

The TTO is up-to-date and has been clinically checked by pharmacy

Any PRN insulin prescribed is still needed and has clear instructions

Double-check if the patient can administer their insulin

Any dose changes have been explained to the patient/family/carer and have been documented on the TTO

If insulin is new or has been changed (e.g. dose) AND administered by district nurses, refer to the DSN so a plan can be put on Systmone

Make sure the patient only goes home with their currently prescribed insulin (NB: check the fridge)

All the correct equipment and information is supplied (sharps bin. lancets, BC monitors etc.)

The patient has a follow-up plan in the community

PLEASE FILE IN THE PATIENT NOTES AFTER COMPLETION PIOT WIL RUN ON RH2 DURING NOVEMBER 2018

Figure 2 Insulin discharge checklist for nursing staff on the ward, implemented in plan-do-study-act (PDSA) cycle 3. PRN, when required; DSN, Diabetes Specialist Nurse; TTO, discharge prescription; BG, blood glucose; NB, nota bene (note well); RH2, Robert Hadfield 2 ward.

3 weeks, the average compliance with the guidelines increased from $50 \%$ to $99 \%$, and was consistently above $90 \%$ during the final week of data collection (figure 3 ).

Improvements were seen in all aspects of quality measures listed in online supplementary table S2, resulting in clearer and more descriptive discharge prescriptions for insulin. Feedback from ward staff was that the interventions resulted in improved interface communication and safer insulin prescribing for patients being discharged from hospital.

An unintended but predictable consequence of the intervention was a slightly increased time taken for pharmacy to undertake medicines reconciliation on admission and clinically verify insulin discharge prescriptions. This was not unacceptable to pharmacy staff, however, due to the perceived positive impact on patient safety:

I think it will have a positive impact on patient safety, so I think every minute spent on the documentation is worth it!

Pharmacy staff on the ward described a heightened vigilance as a result of the poster, particularly with respect to the inappropriate prescribing of 'when required' insulin on discharge, which they described making interventions in for three patients during the project. This highlighted a further ongoing need to engage with prescribers with respect to this particular quality measure. Medical and nursing staff commented that the posters were attractive and informative, and were positive about their potential impact on patient safety.

\section{LESSONS AND LIMITATIONS}

The project aim was to improve the quality of insulin discharge prescriptions in a way that would be sustainable and transferable to different ward environments. As such, although interventions were implemented and developed on a single ward, they targeted the problems identified at a trust-wide level. The need for change was described pictorially with the use of objective data, which helped to highlight the need for change in a way that did not target or criticise particular staff members, professional groups or ward teams. ${ }^{33}$

A key lesson learnt during the process was the importance of the early and continuous involvement of the multidisciplinary team (both at ward and directorate levels) in designing and modifying interventions. As the changes designed were acceptable to the variety of professionals involved in the discharge process, this helped to

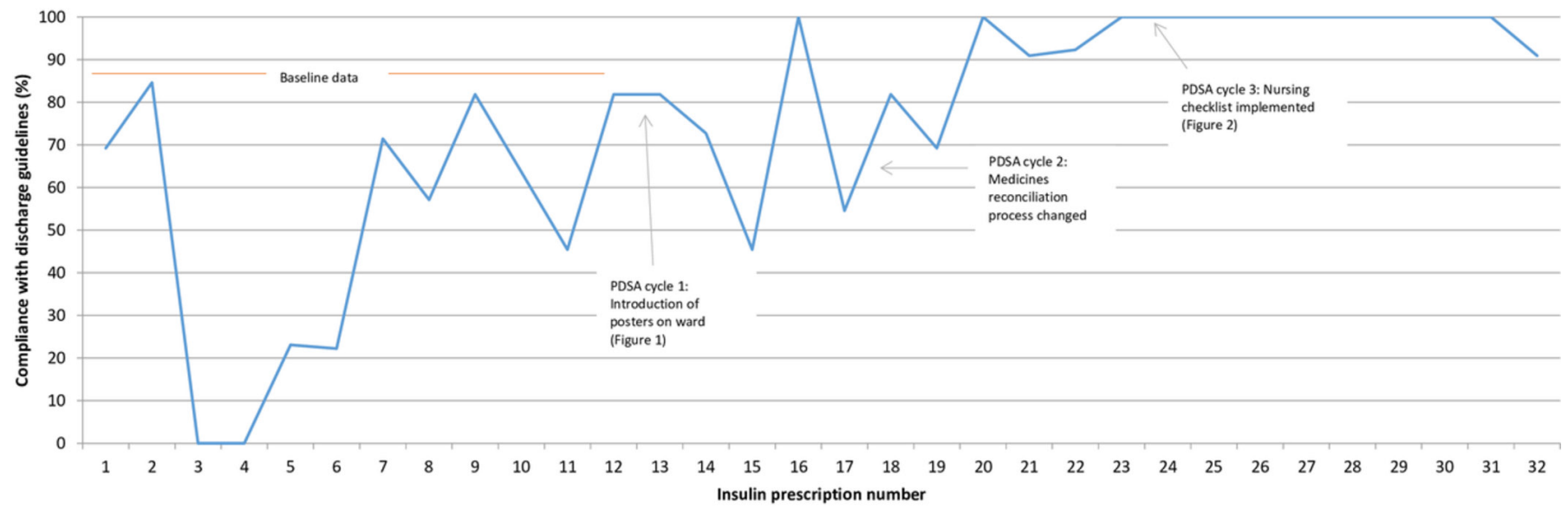

Figure 3 Run chart of insulin discharge prescription guideline compliance throughout the project. PDSA, plan-do-study-act. 
validate the intervention and encourage its viability. The continuous measurement of data and discussion with ward staff to optimise iterative interventions before scaling was key. ${ }^{34}$ As scaling the intervention on a trust-wide level will involve discussion at governance committee level, efforts to engage wider senior staff early on in the intervention design process may be made to increase efficiency in future projects.

Data were collected on discharge prescriptions that had been completed and verified, thereby reflecting the final document received by general practitioners. Any undocumented pharmacist or nursing interventions were therefore not reflected in the quantitative data. Anecdotal qualitative evidence was collected as recommended by Pope $e t a l,{ }^{35}$ despite its limitations and potential biases, to help contextualise and compensate for the limitations in the type of measures being monitored. Balancing measures were also considered, such as the time taken to undertake medicines reconciliation on admission and pharmacist clinical verification.

Future efforts to measure adherence to other areas of the guidance (eg, concerning patient communication and checks made around insulin equipment provision) may be considered to allow a more holistic assessment of discharge quality and success of the intervention. Remote data collection may have minimised bias and observer (Hawthorne) effect, but all ward staff were aware that the success of the intervention would be measured. Future projects may benefit from more rigorous study design, for example, involving a 'control' ward and validation of measurement tools, as described by Portela $e t a l .{ }^{36}$

Ward staff demonstrated positive engagement with the project. This could be due to a combination of factors, including their interest in diabetes care, the high frequency of patients being discharged with insulin from the ward, and the appreciation that discharges from the ward have been problematic in the recent past. Non-diabetes wards that do not discharge many patients on insulin may not be as motivated to engage with QI measures in this area, and the same intervention(s) may not result in the improvements seen in this short project. The presence of diabetes link nurses and diabetes outreach services may, however, offer a potential solution to this. Also, not all discharge prescriptions from the diabetes ward are checked by diabetes pharmacists, particularly if patients are being discharged out of hours. The engagement of the wider pharmacy team will therefore be required for improvements to be consistent.

After further roll-out of the intervention, the re-audit of insulin discharge quality will be required to demonstrate the sustainability of change, and would need to take into account potential positive confounding bias and natural process variation that may affect the results (eg, the change of rotational junior doctor and pharmacy staff on the ward, as well as local ward processes may affect the results independently of the intervention). Statistical analysis would need to be performed on a larger data set obtained over a longer period of time in order to increase the reliability of the results.

\section{CONCLUSION}

It is widely known that insulin is a high-risk drug that is frequently implicated in medication errors in hospital, particularly at the point-of-care transfer. Our aim was to achieve compliance with new consensus-derived, local insulin discharge guidance after the series of interventions, which we achieved in over $90 \%$ by the end of the project. This resulted in clearer communication of insulin information across the interface on discharge prescriptions as well as a reduction in insulin prescribing errors. With appropriate resources, the extra time taken to undertake comprehensive medicines reconciliation on transfer of care would be beneficial in helping to improve the safe use of insulin for patients. Continued measurement of medication safety incidents and errors would be required in order to demonstrate sustainability and success of the intervention.

The focus of the project was adjusted slightly in light of what aspects of discharge quality could be realistically and reliably measured. This included optimising the process of medicines reconciliation using the electronic prescribing system in order to clearly communicate important information regarding insulin therapy. In this sense, the project was useful in identifying what specific aspects of discharge prescriptions could easily be improved, and how EPMA could be better used to optimise communication in order to increase patient safety.

Due to the considerable improvement seen by the interventions in this project, Trust-wide scale up is currently underway. Diabetes link nurses have been informed and engaged in the project and measures have been taken to integrate the interventions into the existing EPMA system and nursing discharge planning processes. Further work is being considered to develop further educational materials for staff that can be integrated into existing systems to promote the safe use of insulin in hospital.

Acknowledgements The authors thank Clare Nelson, Owen Reeve, Dr Fionuala Creagh, Dr Leanne Hunt, Sarah Humphries and all staff on wards Hadfield 1 and 2 who supported and contributed their valuable feedback during this project.

Contributors $\mathrm{AB}$ and $\mathrm{SK}$ conceptualised the project under the academic supervision of JS and GQ. AB and IF designed and iterated the interventions with the help of the project team and ward staff. SK provided supervisory support throughout the project and helped to coordinate the project group. AB and IF undertook data collection, analysis and drafted the manuscript. JS and GQ provided methodological and editorial guidance.

Funding The authors have not declared a specific grant for this research from any funding agency in the public, commercial or not-for-profit sectors.

Competing interests None declared.

Patient consent for publication Not required.

Ethics approval Ethical approval for the project was granted by the University of Bradford (Ref: EC25219) and was not required by STHFT.

Provenance and peer review Not commissioned; externally peer reviewed.

Open access This is an open access article distributed in accordance with the Creative Commons Attribution Non Commercial (CC BY-NC 4.0) license, which 
permits others to distribute, remix, adapt, build upon this work non-commercially, and license their derivative works on different terms, provided the original work is properly cited, appropriate credit is given, any changes made indicated, and the use is non-commercial. See: http://creativecommons.org/licenses/by-nc/4.0/.

\section{REFERENCES}

1. Cousins D, Rosario C, Scarpello J. Insulin, hospitals and harm: a review of patient safety incidents reported to the National patient safety agency. Clin Med 2011;11:28-30.

2. Institute of Safe Medication Practitices. ISMP list of High-Alert medications in acute care settings. Inst Safe Medicat Pract 2011.

3. Ashcroft DM, Lewis PJ, Tully MP, et al. Prevalence, nature, severity and risk factors for prescribing errors in hospital inpatients: prospective study in 20 UK hospitals. Drug Saf 2015;38:833-43.

4. Health and Social Care Information Centre. National diabetes inpatient audit 2017 - national report. Available: https://files.digital. nhs.uk/pdf/s/7/nadia-17-rep.pdf [Accessed 20 Jul 2018].

5. Breuker C, Macioce V, Mura T, et al. Medication errors at hospital admission and discharge in type 1 and 2 diabetes. Diabet Med 2017;34:1742-6.

6. Bain A, Nettleship L, Kavanagh S, et al. Evaluating insulin information provided on discharge summaries in a secondary care hospital in the United Kingdom. J of Pharm Policy and Pract 2017;10.

7. Bain A, Moussallati N, Kavanagh S. Improving insulin prescribing safety on admission with pharmacy intervention. Poster presented at: United Kingdom Clinical Pharmacy Association Conference, 2017.

8. Bain A, Kavanagh S, McCarthy $S$, et al. Assessment of insulin-related knowledge among healthcare professionals in a large teaching hospital in the United Kingdom. Pharmacy 2019;7.

9. Joint British Diabetes Societies for inpatient care. Discharge planning for adult inpatients with diabetes, 2017. Available: http://www. diabetologists-abcd.org.uk/JBDS/JBDS_Discharge_Planning_Final_ 2015.pdf [Accessed 10 Jan 2018].

10. Riordan Ciara O', Delaney T, Grimes T. Exploring discharge prescribing errors and their propagation post-discharge: an observational study. Int J Clin Pharm 2016;38:1172-81.

11. Callen J, Mclntosh J, Li J. Accuracy of medication documentation in hospital discharge summaries: a retrospective analysis of medication transcription errors in manual and electronic discharge summaries. Int J Med Inform 2010;79:58-64.

12. Kripalani S, LeFevre F, Phillips CO, et al. Deficits in communication and information transfer between hospital-based and primary care physicians: implications for patient safety and continuity of care. JAMA 2007;297:831-41.

13. National Institute for Health and Care Excellence. Medicines optimisation: the safe and effective use of medicines to enable the best possible outcomes (NG5). NICE, 2015.

14. 14 NHS England. Patient safety alert stage one: warning risks arising from breakdown and failure to act on communication during handover at the time of discharge from secondary care, 2014. Available: http://www.england.NHS.uk/2014/08/29/psacommunication/ [Accessed 25 Mar 2019].

15. Redmond P, Grimes TC, McDonnell R, et al. Impact of medication reconciliation for improving transitions of care. Cochrane Database Syst Rev 2018;13.
16. National patient safety Agency. National patient safety Agency. safer administration of insulin. NHS, 2010: 14410.

17. National Patient Safety Agency. The adult patient 's passport to safer use of insulin, 2011. Available: http://www.npsa.nhs.uk/corporate/ news/passport-to-safer-use-of-insulin/ [Accessed 13 Jun 2017].

18. Walkers J, Wilcock M. Implementation of the insulin passportapparently going nowhere? Int J Pharm Pract 2014;22:94-5.

19. Bain A, Hasan S, Babar ZUD. Interventions to improve insulin prescribing practice for people with diabetes in hospital: a systematic review. Diabet Med.

20. Hamilton P, Nation M, Penfold S, et al. Reducing insulin prescription errors in hospital: more stick than carrot? Practical Diabetes 2013;30:370-3.

21. Leech NJ, Johnson G, Nayar R, et al. Tackling insulin safety using a multifaceted multidisciplinary regional approach first report from the North East regional insulin safety and knowledge (risk) project, 2013.

22. Health Innovation Network. A guide to improving insulin safety on discharge from hospital for older adults with diabetes, 2017 Available: www.hin-southlondon.org [Accessed 20 Jul 2018].

23. Rushmer R, Voigt D. Measure it, improve it: the safer patients initiative and quality improvement in subcutaneous insulin therapy for hospital in-patients. Diabet Med 2008;25:960-7.

24. Field HT, Woodier N, Clayton J, et al. Use of an educational, audiovisual podcast to maximise safety with variable rate intravenous insulin infusions. BMJ Open Qual 2018;7:e000111.

25. Tully V, Al-Salti S, Arnold A, et al. Interprofessional, student-led intervention to improve insulin prescribing to patients in an acute surgical receiving unit. BMJ Open Qual 2018;7:e000305.

26. Pollack TA, Illuri V, Khorzad R, et al. Risk assessment of the hospital discharge process of high-risk patients with diabetes. BMJ Open Qual 2018;7:e000224.

27. Van Dalen J, Gubbels H, Engel C, et al. Effective poster design. Educ Heal 2002.

28. Atkins $\mathrm{H}$, Boland $\mathrm{A}$, Berrington $\mathrm{R}$, et al. Implementation of ThinkGlucose improves quality of care for inpatients with diabetes. Diabet Med 2013.

29. Ilic D, Rowe N. What is the evidence that poster presentations are effective in promoting knowledge transfer? A state of the art review. Health Info Libr J 2013;30:4-12.

30. Flanagan D, Dhatariya K, Kilvert A, et al. Self-Management of diabetes in hospital: a guideline from the joint British diabetes societies (JBDS) for inpatient care group. Diabet. Med. 2018;35:992-6.

31. Thomassen $\varnothing$., STORESUND A, SØfteland E, et al. The effects of safety checklists in medicine: a systematic review. Acta Anaesthesiol Scand 2014;58:5-18.

32. Levy SM, Senter CE, Hawkins RB, et al. Implementing a surgical checklist: more than checking a box. Surgery 2012;152:331-6.

33. Radhakrishna S. Culture of blame in the National health service; consequences and solutions. Br J Anaesth 2015;115:653-5.

34. Maher L, Gustafson D, Evans A. Nhs sustainability model Institute for innovation and improvement, 2010. Available: https://www.england. nhs.uk/improvement-hub/wp-content/uploads/sites/44/2017/11/ NHS-Sustainability-Model-2010.pdf [Accessed 20 Mar 2018].

35. Pope C, van Royen P, Baker R. Qualitative methods in research on healthcare quality. Qual Saf Health Care 2002;11:148-52.

36. Portela MC, Pronovost PJ, Woodcock T, et al. How to study improvement interventions: a brief overview of possible study types: Table 1. BMJ Qual Saf 2015;24:325-36. 